\title{
Effect of Two Strains of Probiotics on the Antioxidant Capacity, Oxidative Stress, and Immune Responses of Salmonella-Challenged Broilers
}

-Author(s)

\section{Abudabos AM \\ Alyemni $\mathrm{AH}^{\prime \prime}$ \\ Zakaria $\mathrm{HAH}^{\prime \prime}$}

Department of Animal Production, College of Food and Agriculture Sciences, King Saud University, P. O. Box 2460, Riyadh 11451, Kingdom of Saudi Arabia.

" ARASCO for Feed, P.O Box 53845, Riyadh 11593, Kingdom of Saudi Arabia.

III Animal Production Department, The University of Jordan, Amman, Jordan

\section{Mail Address}

Corresponding author e-mail address Dr. Alaeldein M. Abudabos

Department of Animal Production, College of Food and Agriculture Sciences, King Saud University, P. O. Box 2460, Riyadh

11451, Saudi Arabia

Phone: 00966597634578

Fax: 0096614678474

Email: alabudabos@gmail.com

\section{-Keywords}

Immune responses, Probiotics, Oxidative stress, Salmonella enterica, Total antioxidant capacity (TAC).

\section{ABSTRACT}

The present study aimed at evaluating the effect of two strains of probiotics (Bacillus subtilis PB6 and Bacillus Cereus var. toyoi) on selected indicators of the antioxidant status and immune responses of broilers before and after challenge with Salmonella enterica subsp. Typhimurium (S. typhimurium). Birds were distributed into the following five treatments: negative control $(C T L-)$; negative control+S. typhimurium $(C T L+)$; antibiotic $+S$. typhimurium, $(A B)$; Toyocerin $\AA+S$. typhimurium (TOYO); and CloSTAT ${ }^{\mathrm{TM}}+$ S. typhimurium (CLOS). Birds in all treatments, except for $\mathrm{CTL}-$, were orally challenged with $3 \times 10^{9}$ CFUS. typhimurium at 16 days of age. The results showed that total antioxidant capacity (TAC), superoxide dismutase activity (SOD), and hydrogen peroxide $\left(\mathrm{H}_{2} \mathrm{O}_{2}\right)$ values were similar among all groups. The antibody titers against $S$. typhimurium and Newcastle disease (ND) were not affected by the treatments $(p>0.05)$. However, $S$. typhimurium and ND titers were influenced by the interaction between week and treatment $(p<0.01, p<0.05$, respectively) and by week $(p<0.001$, $p<0.001$, respectively). In conclusion, $S$. typhimurium challenge had an impact on anti-Salmonella and NC titers. Both probiotics did not affect the broilers' antioxidant capacity.

\section{INTRODUCTION}

Salmonella enterica serovar Typhimurium (S. typhimurium) is an enteric bacterium that can colonize chickens; it is a common serovar causing salmonellosis in broiler chickens (Ribeiro et al., 2007).

Probiotics have been reported to prevent the colonization of the gut by pathogenic bacteria like Clostridium perfringens and Salmonella through the mechanism of competitive exclusion (Teo and Tan, 2007). A Bacillus subtilis-based probiotic was capable of producing an antimicrobial factor against many bacteria (Yurong et al., 2005). Other reports showed that a probiotic based on Bacillus Cereus var. toyoi reduced the prevalence of Salmonella in poultry (Vilà et al., 2005).

Sahin et al. (2004) reported Japanese quails submitted to stress presented higher oxidative stress and lower antioxidant status than non-stressed ones. Probiotic supplementation was shown to modulate the dynamics of oxidants and antioxidants in the body. For example, probiotics based on Bifidobacterium and Lactobacillus showed good antioxidant effect by inhibiting lipid peroxidation in vitro using intact cells and intracellular cell-free extracts (Lin and Yen, 1999). Similarly, probiotics have been shown to increase antioxidant levels and reduce glutathione concentrations and intestinal oxidative injury (Yadav et al., 2007). Sohail et al. (2011) demonstrated that a probiotic reduced the total antioxidant concentration. In another study, rats fed a probiotic showed increased SOD and glutathione peroxidase concentrations (D'Souza et al., 2010). 
Probiotics supplementation was reported to enhance disease resistance and improve the immune response of broiler chickens (Huang et al., 2004). A study by Haghighi et al. (2006)suggested that probiotics have an immune-boosting role due to enhanced induction of natural antibodies in chickens.

Therefore, the objective of the present study was to evaluate the effect of two strains of probiotics (Bacillus subtilis PB6 and Bacillus Cereus var. toyoi) on selected indicators of antioxidant status and immune responses of broilers before and after challenge with Salmonella enterica subsp. Typhimurium (S. typhimurium).

\section{MATERIALS AND METHODS}

\section{Animals and treatments}

Two hundred day-old Cobb 500 broilers, vaccinated against Newcastle disease, infectious bronchitis and
Marek's disease, were allotted to 50 experimental cages (four birds per cage) in a controlled-temperature environment, under 24-h lighting, and were offered feed and water ad libitum. The experiment was carried out from day one to day 42 .

A two-phase feeding program was applied (starter: 0-14 days of age; finisher: 15-42 days of age). Diet formulation is shown in Table 1.Five treatments were tested, each with ten replicates as follows: T1 = control group, non-medicated diet (CTL-); $\mathrm{T} 2=$ nonmedicated diet and challenged with $S$. typhimurium $(\mathrm{CTL}+) ; \mathrm{T} 3=$ antibiotic $(0.05$ gneoxyval $/ \mathrm{kg}$ diet $)$ and challenged with $S$. typhimurium, (AB); $\mathrm{T} 4=$ probiotic (1 $\mathrm{g} / \mathrm{kg}$ diet Toyocerin $\AA$ ) and challenged with $S$. typhimurium (TOYO); and T5 = probiotic (1 g/kg diet ClOSTAT $^{\text {TM }}$ ) and challenged with $S$. typhimurium (CLOS). Toyocerin $®$ consists of $1 \times 10^{9} \mathrm{CFU} / \mathrm{g}$ viable spores of a microorganism Bacillus Cereus var. toyoi

Table 1 - Dietary ingredients and chemical composition of starter and finisher diets

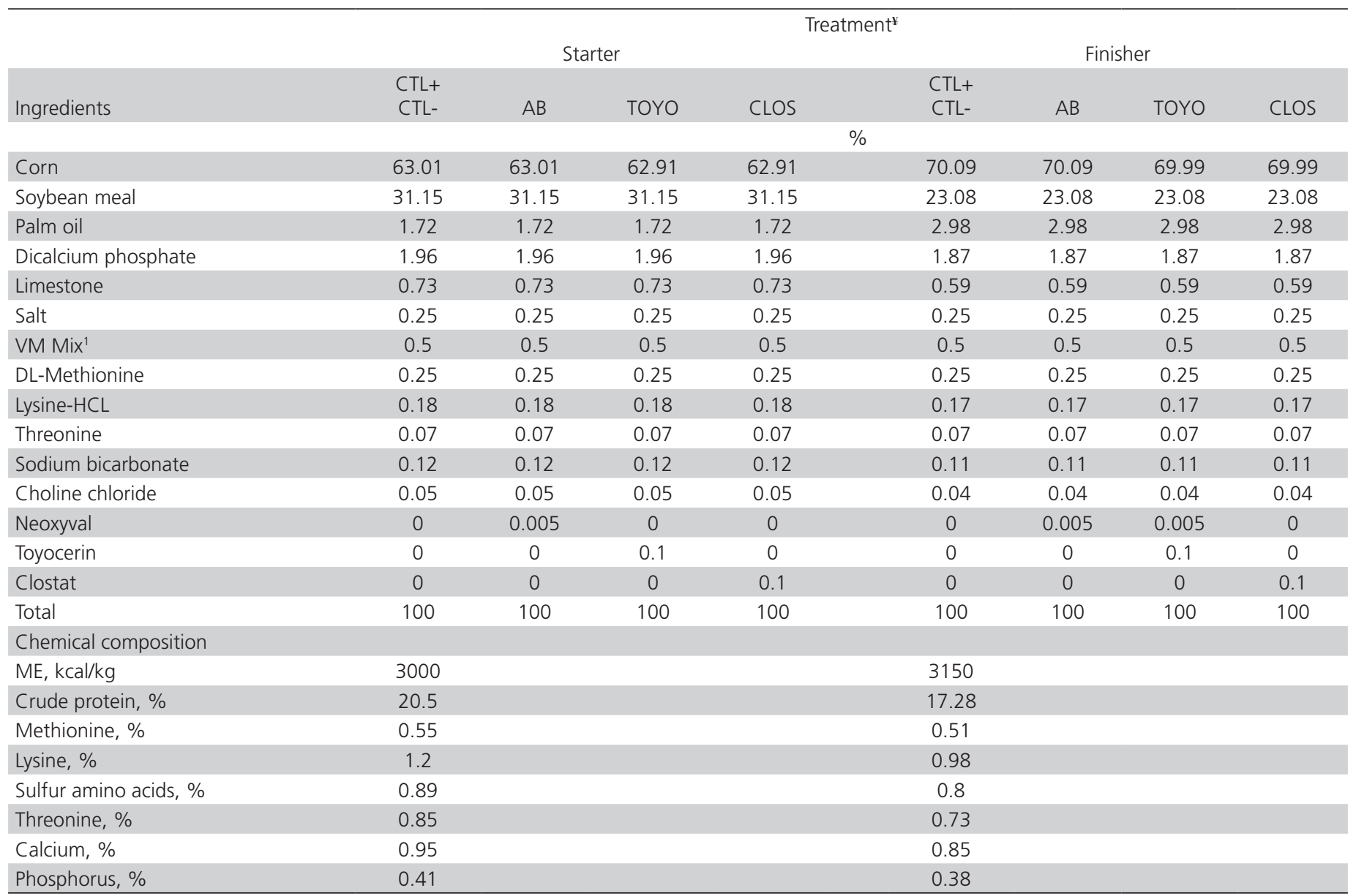

'Vitamin-mineral premix contains in the following per $\mathrm{kg}$ : vitamin A, $2400000 \mathrm{IU}$; vitamin D, $1000000 \mathrm{IU}$; vitamin E, $16000 \mathrm{IU}$; vitamin K, $800 \mathrm{mg}$; vitamin B1, $600 \mathrm{mg}$; vitamin B, 1600 mg; vitamin $B_{6^{\prime}} 1000 \mathrm{mg}$; vitamin $B_{12}, 6$ mg; niacin, 8000 mg; folic acid, 400 mg; pantothenic acid, 3000 mg; biotin 40 mg; antioxidant, 3000 mg; cobalt, 80 mg; copper, 2000 $\mathrm{mg}$; iodine, 400; iron, $1200 \mathrm{mg}$; manganese, $18000 \mathrm{mg}$; selenium, $60 \mathrm{mg}$, and zinc, $14000 \mathrm{mg}$.

${ }^{*} \mathrm{~T} 1=$ control group, non-medicated diet, unchallenged birds, $(\mathrm{CLT}+) ; \mathrm{T} 2=$ non-medicated diet + bacterial challenge (CLT); $\mathrm{T} 3=$ medicated diet with neoxyval $(0.05 \mathrm{~g} / \mathrm{kg}$ diet $)+$ bacterial challenge (AB); T4= toyocerin (1 $\mathrm{g} / \mathrm{kg}$ diet) + bacterial challenge (TOYO); and T5=CloSTAT (1 g/kg diet) + bacterial challenge (COLS). 
(Rubinum, Spain). CloSTAT ${ }^{\mathrm{TM}}$ contains $2 \times 10^{7} \mathrm{CFU} / \mathrm{g}$ of Bacillus subtilis PB6 (CloSTAT, Kemin Industries Inc., Des Moines, IA). At day 16 of age, all birds except for those in the CTL-treatment were orally inoculated with $3 \times 10^{9}$ CFU of Salmonella Typhimurium ATCC13311,according to the procedure described by Abudabos et al. (2014).

\section{Measurements}

Serum total antioxidant capacity (TAC), superoxide dismutase activity (SOD), and hydrogen peroxide $\left(\mathrm{H}_{2} \mathrm{O}_{2}\right)$ levels were measured using commercial kits (Cell Biolabs, Inc., San Diego, USA). For this purpose, blood samples were collected from 10randomly selected birds per treatment via brachial venipuncture and poured into plain tubes on day 42 . Samples were centrifuged at $5^{\circ} \mathrm{C}$ and 3,000 rpm for $10 \mathrm{~min}$, and then the serum was harvested.

Anti-S. typhimurium and Newcastle disease virus (ND) antibodies were titered in the serum from 10birds per treatment before (at 7 days of age) and after (at 21, 28 and 35 days)S. typhimurium challenge.Anti-S. typhimurium antibody titers were determined using a commercial ELISA kit (BioChek, London, UK). Microtiter plates pre-coated with inactivated S. typhimurium antigen were used. Serum samples were diluted $(1: 100)$ in diluent reagent and added to the wells of the microtiter plates to allow the formation of antigen-antibody complexes in the presence of anti-S. typhimurium antibodies. Anti-chicken IgG labelled with the enzyme alkaline phosphatase was then added to the wells. The chromogen added was para-nitrophenylphosphate (pNPP), and absorbance was read at $405 \mathrm{~nm}$ by using a microtiter plate reader.

A commercial Newcastle disease virus antibody test kit (IDEXX Laboratories, Inc., Westbrook, Maine, USA) was used for the detection of antibodies against ND. Serum samples were diluted (1:500) with diluent reagent and $100 \mu \mathrm{L}$ of samples were added to the microtiter wells. Plates were incubated for 30 minutes at $18-26 \mathrm{C}^{\circ}$, and then washed with $350 \mu \mathrm{L}$ of distilled water, $100 \mu \mathrm{L}$ of conjugate were dispensed into each well, and after incubation for 30 minutes at 18-26 $\mathrm{C}^{\circ}$, $100 \mu \mathrm{L}$ of TMB substrate solution were dispensed into each well and $100 \mu \mathrm{L}$ of stop solution were dispensed to stop the reaction. Absorbance was read at $650 \mathrm{~nm}$.

On day 42, 10 birds per treatment were slaughtered, and their liver, bursa, spleen, and thymus were collected and weighed. Their weights relative to carcass weight were calculated and expressed as percentages.

\section{Statistical Analysis}

Data were analyzed according to a completely randomized block design, with five treatments of 10 replicates each. The general linear model procedure of SAS (version 9.1. SAS Institute, Cary, NC) was used. The effects of week of collection, treatment and week $x$ treatment interaction were tested. Means showing significant differences in the analysis of variance were compared using the PDIFF option. The overall statistical significance level was set at $p<0.05$. All values were expressed as statistical means \pm standard error of the mean (SEM).

\section{RESULTS AND DISCUSSION}

Table 2 shows the serum TAC, SOD and $\mathrm{H}_{2} \mathrm{O}_{2}$ results of the birds submitted to the experimental treatments. Serum TAC, SOD, and $\mathrm{H}_{2} \mathrm{O}_{2}$ values were similar ( $p>0.05$ ) among all groups, suggesting that neither $S$. typhimurium challenge nor probiotics had an impact on total antioxidant capacity or oxidative stress. These results agree with Erdoğan et al. (2010),who concluded that the probiotics tested did not promote any significant improvement in the oxidant/antioxidant status in broilers. On the other hand, several other reports provided evidence that probiotics positively modulate the dynamics of oxidants and antioxidants in the body of chickens, Japanese quails, and rats (Lin and Yen, 1999; Sahin et al., 2004; Yadav et al., 2007; Sohail et al., 2011). This difference may be explained by several factors, such as probiotic strain and challenge type. Lin and Yen (1999) hypothesized that beneficial intestinal bacteria produce certain factors that have the ability to chelate free radicals, capturing ROS and inhibitingtheir cytotoxic activity.

Table 2 - Total antioxidant capacity and oxidative stress (SOD and $\mathrm{H}_{2} \mathrm{O}_{2}$ levels) in serum of broiler chickens fed the experimental diets.

\begin{tabular}{lccc}
\hline Treatment & TAC & SOD & $\mathrm{H}_{2} \mathrm{O}_{2}$ \\
& $\mathrm{Mm}$ & $\mu \mathrm{L}$ & $\mu \mathrm{M}$ \\
CTL+ & 0.54 & 0.17 & 78.78 \\
CTL- & 0.63 & 0.16 & 71.80 \\
$\mathrm{AB}$ & 0.61 & 0.29 & 68.44 \\
TOYO & 0.59 & 0.24 & 91.98 \\
CLOS & 0.57 & 0.32 & 75.10 \\
& & & \\
SEM & 0.028 & 0.04 & 10.39 \\
P value & $\mathrm{NS}$ & $\mathrm{NS}$ & $\mathrm{NS}$ \\
\hline
\end{tabular}

The mean corresponds to 10 birds per treatment.

Oxidative stress can be defined as a physiological disorder where there is an imbalance between concentrations of reactive oxygen species (ROS), such 
as hydrogen peroxide $\left(\mathrm{H}_{2} \mathrm{O}_{2}\right)$, and an antioxidant. Sahin et al. (2002) reported that low endogenous antioxidant production is the causative factor of oxidative stress in birds. Excessive ROS accumulation leads to cell injury, including damage to the DNA, proteins, and lipid membranes. Generally, ROS are continually produced by aerobic cells and are instantly removed by endogenous antioxidant scavengers. It is established that changes in the activity of several antioxidant enzymes can be used to assess the level of oxidative stress and total antioxidant status in birds. TAC accurately reflects the antioxidative status of the organism. SOD catalyzes the dismutation of the superoxide anion into hydrogen peroxide, and molecular oxygen is considered as one of the most potent antioxidant. Hydrogen peroxide $\left(\mathrm{H}_{2} \mathrm{O}_{2}\right)$ is one of the most well-documented ROS produced under oxidative stress. It is a toxic product of normal aerobic metabolism and pathogenic ROS production involving oxidase and superoxide dismutase reactions. Under normal physiological conditions, cellular ROS generation is counter balanced by the action cellular antioxidant enzymes and redox molecules.

The results for anti- $S$. typhimurium responses in the different treatments are presented in Table 3. There was a significant treatment ${ }^{*}$ week interaction $(p<0.01)$. The $S$. typhimurium titer continued to increase in birds in treatments $C T L+, A B$, and TOYO from week one through week five. In the CTL- birds, S. typhimurium titer increased from week 1 to week 3 , then decreased in week 4 and increased again in week $5(0.6,1.17$, 1.02 and 2.38 , for weeks $1,3,4$, and 5 , respectively). In the birds in the CLOS group, the titer increased until week 4 , then decreased in week $5(1.09,1.42$, 1.62 and 0.92 , for weeks $1,3,4$, and 5 , respectively). On the other hand, week showed a significant effect on anti-S. typhimurium titer, which increased from week 1 to week $5(0.73,1.85$, respectively; $p<0.001)$, while treatment had no significant effect on anti-S. typhimurium titers $(p>0.05)$. The beneficial effects of probiotics of boosting the immune system of broilers is due to enhanced induction of natural antibodies, as previously reported (Haghighi et al., 2006).

The Newcastle disease (NC) titer results are shown in Table 4. Treatment showed no effect on NC titer; however, there was a significant treatment*week interaction $(p<0.05)$ and a strong effect of week $(p<0.001)$. Birds in all treatments presented lower NC titers after S. typhimurium challenge (week 3) as compared to week 1 (before the challenge). However, the response after week 3 varied according to the treatment. For example, the NC titer of birds in groups
Table 3 - Serum anti-Salmonella antibody titers according to treatment and week

\begin{tabular}{|c|c|c|}
\hline Treatment & & Titer \\
\hline CTL+ & & 1.29 \\
\hline CTL- & & 1.33 \\
\hline$A B$ & & 1.25 \\
\hline TOYO & & 1.31 \\
\hline CLOS & & 1.26 \\
\hline SEM & & 0.11 \\
\hline$P$ value & & NS \\
\hline Week & Challenge & \\
\hline 1 & Before & $0.73^{d}$ \\
\hline 3 & After & $1.14^{c}$ \\
\hline 4 & After & $1.45^{b}$ \\
\hline 5 & After & $1.85^{a}$ \\
\hline SEM & & 0.09 \\
\hline$p$ value & & 0.001 \\
\hline Week & Treatment & \\
\hline 1 & CTL+ & 0.6 \\
\hline 1 & CTL- & 0.69 \\
\hline 1 & $A B$ & 0.48 \\
\hline 1 & TOYO & 0.77 \\
\hline 1 & CLOS & 1.17 \\
\hline 3 & CTL+ & 1.18 \\
\hline 3 & CTL- & 1.12 \\
\hline 3 & $A B$ & 1.02 \\
\hline 3 & TOYO & 0.98 \\
\hline 3 & CLOS & 1.42 \\
\hline 4 & CTL+ & 1.02 \\
\hline 4 & CTL- & 1.65 \\
\hline 4 & $A B$ & 1.39 \\
\hline 4 & TOYO & 1.55 \\
\hline 4 & CLOS & 1.62 \\
\hline 5 & CTL+ & 2.38 \\
\hline 5 & CTL- & 1.86 \\
\hline 5 & $A B$ & 2.13 \\
\hline 5 & TOYO & 1.93 \\
\hline 5 & CLOS & 0.93 \\
\hline SEM & & 0.22 \\
\hline Treatment* week & & $\star *$ \\
\hline
\end{tabular}

CTL-, CTL+, and TOYO continued to increase after week 3 , but not in the birds fed AB and CLOS. Sadeghl et al. (2013) found that S. typhimurium-infected birds had higher antibody titers in response to NC vaccination when compared to uninfected birds. Holt et al. (1999) concluded that broilers exposed to Salmonella at a young age may remain infected upon maturity and do not develop significant immunity against Salmonella. Seo et al. (2000) evaluated the effects of the challenge with Salmonella Enteritidis, enrofloxacin and/or normal avian gut flora in layers. They found that antibodies titers (lgG) were similar among treatments at two days 
Table 4 - Serum anti-Newcastle disease titers according to treatment and week

\begin{tabular}{|c|c|c|}
\hline Treatment & & Titer \\
\hline CTL+ & & 2.4 \\
\hline CTL- & & 2.28 \\
\hline$A B$ & & 2.29 \\
\hline TOYO & & 2.27 \\
\hline CLOS & & 2.47 \\
\hline SEM & & 0.11 \\
\hline$P$ value & & NS \\
\hline Week & Challenge & \\
\hline 1 & Before & $2.67^{a}$ \\
\hline 3 & After & $1.75^{b}$ \\
\hline 4 & After & $2.44^{\mathrm{a}}$ \\
\hline 5 & After & $2.42^{\mathrm{a}}$ \\
\hline SEM & & 0.1 \\
\hline$P$ value & & 0.0001 \\
\hline Week & Treatment & \\
\hline 1 & CTL+ & 2.77 \\
\hline 1 & CTL- & 2.6 \\
\hline 1 & $A B$ & 2.78 \\
\hline 1 & TOYO & 2.63 \\
\hline 1 & CLOS & 2.45 \\
\hline 3 & CTL+ & 1.94 \\
\hline 3 & CTL- & 1.88 \\
\hline 3 & $A B$ & 2.17 \\
\hline 3 & TOYO & 1.47 \\
\hline 3 & CLOS & 1.18 \\
\hline 4 & CTL+ & 2.43 \\
\hline 4 & CTL- & 2.09 \\
\hline 4 & $A B$ & 2.27 \\
\hline 4 & TOYO & 2.47 \\
\hline 4 & CLOS & 2.93 \\
\hline 5 & CTL+ & 2.46 \\
\hline 5 & CTL- & 2.55 \\
\hline 5 & $A B$ & 1.83 \\
\hline 5 & TOYO & 2.50 \\
\hline 5 & CLOS & 2.75 \\
\hline SEM & & 0.22 \\
\hline Treatment*week & & * \\
\hline
\end{tabular}

of age and only $8 \%$ of samples presented detectable anti-SE antibody titers after four weeks. Ribeiro et al. (2007) found that a probiotic had no effect on the production of anti-Salmonella Enteritidis antibodies in Salmonella-challenged birds.

Also, NC titers were high during week 1 (before the challenge) and then significantly dropped in week 3after the challenge ( 2.67 vs. 1.75 , respectively) $(p<0.05)$. In weeks 4 and 5 , the titers increased to a level similar to those determined in week 1 ( $p>0.05)$. Sedeghi et al. (2013) concluded that Salmonella challenge decreased antibody titers against ND.
Average organ relative weights are documented in Table 5. Treatments did not influence liver, bursa, spleen or thymus relative weights $(p>0.05)$. Peyer's patches, cecal tonsils, and the bursa of Fabricius are sites of lymphoid tissue accumulation in poultry. These organs capture antigens that circulate in the digestive tract and stimulate the production of $B$ and $T$ immune cells. Changes in immune tissue weight affect immune cell phenotypes, immune cell proliferation, and antibody production. Sedeghi et al. (2013) reported that Salmonella challenge reduced the relative weights of immune organs as well as antibody titers against Newcastle. Ciraci et al. (2010) evaluated the effects of Salmonella on the global gene expression of chicken immune tissues or cells. Coble et al. (2013) evaluated transcriptonic profiles from livers of old 10 days old broilers which were infected with $S$. enteritidis and reported a down regulation of genes that function in metabolic pathways, inflammation and mitochondria apoptosis.

Table 5 - Relative organ weight according to treatment.

\begin{tabular}{lllll}
\hline Treatment & Liver & Bursa & Spleen & Thymus \\
$\%$ & $\%$ & $\%$ & $\%$ \\
CTL+ & 2.91 & 0.22 & 0.13 & 0.54 \\
CTL- & 2.62 & 0.19 & 0.13 & 0.59 \\
AB & 2.91 & 0.18 & 0.13 & 0.54 \\
TOYO & 2.71 & 0.20 & 0.13 & 0.68 \\
CLOS & 2.87 & 0.24 & 0.15 & 0.60 \\
& & & & \\
SEM & \pm & 0.02 & 0.01 & 0.06 \\
p value & 0.14 & NS & NS & NS \\
\hline & NS & & &
\end{tabular}

\section{CONCLUSIONS}

Neither S. typhimurium challenge nor probiotics affected broilers' total antioxidant capacity or oxidative stress. Antibody titers against $S$. typhimurium and Newcastle disease (ND) were not affected by treatments. However, both measurements showed a significant week $\mathrm{x}$ treatment interaction.

\section{ACKNOWLEDGEMENTS}

The authors would like to extend their sincere appreciation to the Deanship of Scientific Research at King Saud University for its funding of this research through the Research Group Project No. RGP-VPP-273.

\section{REFERENCES}

Abudabos AM, Yehia HM, Alotybi MN, Garelnabi AR, Alyemni AH. Effects of direct-fed microbial on broiler performance and susceptibility to oral salmonella enteritidis challenge. Journal of Food, Agriculture and Environment 2014;12(2):30-34. 
Ciraci C, Tuggle CK, Wannemuehler MJ, Nettleton D, Lamont SJ. Unique genome-wide transcriptome profiles of chicken macrophages exposed to Salmonella-derived endotoxin. BMC Genomics 2010;11:545.

Coble DJ, Sandford EE, Ji T, Abernathy J, Fleming D, Zhou H, et al. Impacts of Salmonella enteritidis infection on liver transcriptome in broilers. Genesis 2013;51:357-364

D'Souza A, Fordjour L, Ahmad A, Cai C, Kumar D, Valencia G, et al. Effects of probiotics, prebiotics, and synbiotics on messenger RNA expression of caveolin-1, NOS, and genes regulating oxidative stress in the terminal ileum of formula-fed neonatal rats. Pediatric Research 2010;67:526-531.

Erdoğan Z, Erdoğan S, Aslantaş Ö, Çelik S. Effects of dietary supplementation of synbiotics and phytobiotics on performance, caecal coliform population and some oxidant/antioxidant parameters of broilers. Journal of Animal Physiology and Animal Nutrrition 2010;94:e40-e48.

Haghighi HR, Gong J, Gyles CL, Hayes MA, Zhou H, Sanei B, et al. Probiotics stimulate production of natural antibodies in chickens. Clinical Vaccine Immunology 2006;13:975-980.

Holt PS, Gast RK, Porter Jr RE, Stone HD. Hyporesponsiveness of the systemic and mucosal humoral immune systems in chickens infected with Salmonella enterica serovar Enteritidis at one day of age. Poultry Science 1999;78(11):1510-1517.

Huang MK, Choi YJ, Houde R, LeeJW, Lee B, Zhao X. Effects of Lactobacilli and an acidophilic fungus on the production performance and immune responses in broiler chickens. Poultry Science 2004;83(5):788-795.

Lin MY, Yen CL. Antioxidative ability of lactic acid bacteria. Journal of Agriculture and Food Chemistry 1999;47:1460-1466.

Ribeiro AML, Vogt LK, Canal CW, Cardoso MR, Labres RV, Streck AF, et al. Effects of prebiotics and probiotics on the colonization and immune response of broiler chickens challenged with Salmonella Enteritidis. Brazilian Journal of Poultry Science 2007;9(3):193 -200.
Sadeghi AS, Mohammadi A, Shawrang P, Aminafshar M. Immune responses to dietary inclusion of prebiotic-based mannan-oligosaccharide and $\beta$-glucan in broiler chicks challenged with Salmonella enteritidis. Turkish Journal of Veterinary and Animal Science 2013;37:206-213

Sahin K, Kucuk O, Sahin N, Sari M. Effects of vitamin C and vitamin E on lipid peroxidation status, some serum hormone, melite, and mineral concentrations of Japanese quails reared under heat stress $\left(34^{\circ} \mathrm{C}\right.$ ). International Journal Vitamin and Nutrition Research 2002;72:91-100.

Seo KH, Holt PS, Gast RK, Hofacret CL. Elimination of early Salmonella enteritidis infection after treatment with competitive-exclusion culture and enrofloxacin in experimentally infected chicks. Poultry Science 2000;79:1408-1413.

Sohail MU, Rahman ZU, ljaz A, Yousaf MS, Ashraf K, Yaqub T, et al. Single or combined effects of mannan-oligosaccharides and probiotic supplements on the total oxidants, total antioxidants, enzymatic antioxidants, liver enzymes, and serum trace minerals in cyclic heatstressed broilers. Poultry Science 2011;90(11):2573-2577.

Teo AYL, Tan HM. Evaluation of the performance and Intestinal gut microflora of broilers fed on corn-soy diets supplemented with Bacillus subtilis PB6 (CloSTAT). Journal of Applied PoultryResearch 2007;16:296-303

Vilà B, Fontgibell A, Badiola I, Esteve-Garcia E, Jiménez G, Castillo M, et al. Reduction of Salmonella enterica var. Enteritidis colonization and invasion by Bacillus cereus var. toyoi inclusion in poultry feeds. Poultry Science 2009;88(5):975-979.

Yadav $\mathrm{H}$, Jain S, Sinha PR. Antidiabetic effect of probiotic dahi containing Lactobacillus acidophilus and Lactobacillus casei in high fructose fed rats. Nutrition 2007;23:62-68.

Yurong Y, Ruiping S, Shimin Z, Yibao J. Effect of probiotics on intestinal mucosal immunity and ultrastructure of cecal tonsils of chickens. Archives of Animal Nutrition 2005;59:237-246. 